\title{
Time Resolved Full-Annulus Computations of a Turbine with Inhomogeneous Inlet Conditions
}

\author{
Rafael R. Adamczuk and Joerg R. Seume \\ Institute of Turbomachinery and Fluid Dynamics \\ Leibniz Universitaet Hannover \\ Appelstraße 9, 30167 Hannover, GERMANY
}

\begin{abstract}
Jet engine spare parts are expensive. Hence, for economic reasons, as many components as possible should be repaired. For planning the repair or regeneration process, defects must be identified early. The present paper explores whether the detection and examination of inhomogeneities in the temperature distribution in an exhaust gas stream of a jet engine can be used for the identification and localization of defects before engine disassembly. In order to be able to assign the defects to the resulting temperature non-uniformity in the exhaust gas, the mixing characteristics of the temperature inhomogeneities in the turbine caused by the defects are examined. A three-dimensional unsteady CFD-simulation of a whole turbine with a complex temperature distribution at the inlet is carried out. The simulations show, that the temperature inhomogeneities are barely mixed out with the surrounding flow. To describe the mixing effects a coefficient (turbine mixing factor) is derived. The factor enables an integral assessment of the mixing behaviour of inhomogeneities within and outside of turbine stages.
\end{abstract}

\section{NOMENCLATURE}

TMF $\quad[\mathrm{K}] \quad$ turbine mixing factor

$\mathrm{T} \quad[\mathrm{K}] \quad$ temperature

$\mathrm{S} \quad[\mathrm{J} / \mathrm{K}] \quad$ entropy

$\mathrm{R} \quad[-] \quad$ temperature ratio

A...G [-] definition of cold streaks

$\zeta \quad[-] \quad$ total pressure loss coefficient

$\mathrm{r} \quad[\mathrm{m}] \quad$ radius

$\mathrm{y}^{+} \quad[-] \quad$ nondimensional wall distance

$\mathrm{V} \quad[-] \quad$ stator vane

B [-] rotor blade

EGV [-] exit guide vane

$\Theta \quad\left[{ }^{\circ}\right] \quad$ angle in circumferential direction

\section{Subscripts}

$\begin{array}{ll}0 & \text { stage inlet } \\ 1 & \text { stage outlet } \\ \infty & \begin{array}{l}\text { revered to the surrounding unaffected flow } \\ \text { of a cold streak }\end{array}\end{array}$

Presented at International Gas Turbine Congress 2011 Osaka,

November 13-18, Osaka, Japan, IGTC2011-0175

Review completed March 30, 2012

$\begin{array}{ll}50 \% & \text { reveres to the midspan of a stage } \\ \text { avg } & \text { average } \\ \text { tot } & \text { total } \\ \text { st } & \text { static } \\ \text { cs } & \text { cold streak } \\ \text { rel } & \text { relative to the maximal value }\end{array}$

\section{INTRODUCTION}

The investigation of new ways in the regeneration of a modern jet engine used in civil aviation has the goal not only to restore but also to improve the functional characteristics of an engine. The Collaborative Research Centre (CRC) 871 "Regeneration of Complex Capital Goods" develops scientific basics for the maintenance of complex capital goods. The aim is to maintain or to reprocess as many components as possible and hence to regenerate or even improve the functional characteristics of the capital good. Due to the technical complexity and continuous growing requirements regarding the economic efficiency and reliability, modern jet engines have been chosen exemplarily as the complex capital good investigated in the CRC 871. The high requirements for the regeneration-process demand an increase of the life time and a precise maintenance which detects and repairs smaller failures which would cause, in case of further operation, serious malfunctions.

One main goal is to develop a quicker and accurate assessment of the condition of a jet engine which detects the location and character of defined defects in the hot gas path before the disassembling of the jet engine. As every defect in the hot gas path has a direct influence on the local temperature, the identification, localization, and quantification of inhomogeneities in the temperature distribution of an exhaust gas stream can be used for the identification and localization of these defects. The optical measurements of the temperature field are carried out by a tomographic configuration of the Background Oriented Schlieren (BOS) method (see [1]) as presented in [2] and applied to linear cascades in [3]. To be able to assign defects to the resulting temperature inhomogeneity in the exhaust gas stream, detailed knowledge about the mixing characteristics of the cold streaks within the turbine is necessary. This could be achieved by implementing specific defects in a jet engine and conducting measurements with BOS at the jet engine outlet to resolve the density gradients. As this approach is very complex and cost-intensive, CFD-simulations of the flow in a turbine shall be carried out. The aim is to understand the general mixing characteristics of temperature inhomogeneities in turbines. Furthermore the change of the circumferential position shall be examined in order to be able to draw a conclusion on the circumferential and axial position of the defect. As the extent of the circumferential deflection of a cold streak over the whole turbine is unknown and 
several different temperature inhomogeneities shall be examined a complex temperature distribution at the inlet of a turbine is required. Hence a three-dimensional unsteady CFD-simulation of the whole turbine is necessary.

Until now only few time resolved full annulus simulations of axial turbomachines have been carried out. Furthermore most of the simulations were applied for axial compressors. In [4] and [5] transient simulations of a 15 -stage axial compressor were described However, computations with only five stages with 32 million nodes were achieved so far. Herrick et al. [6] performed an unsteady full annulus simulation of one transonic axial compressor stage to validate a Navier-Stokes CFD-code. Im et al. [7] investigated unsteady stall flows of a full annulus transonic rotor. In [8] unsteady flow effects in an axial compressor were investigated. However, the investigation was limited to the first two stages of the compressor. Hence the computations presented in this paper are first of its kind, as time-resolved computations of a whole turbine of a jet engine have, to the authors knowledge, not yet been carried out. Furthermore current fields of research are combustor hot streaks and their effect on the following turbine stages [9]-[11]. Hence, inverse conclusions from the temperature difference and position of an inhomogenity at the exit of a turbine to a possible location within the hot gas streak have not yet been investigated. In addition, mixing effects over a whole turbine have not been examined. In this paper the mixing behaviour of defects which cause decreased local temperatures are investigated. In a first step a low pressure turbine of a modern jet engine is used so that blade cooling effects do not need to be considered.

\section{NUMERICAL METHODS}

The computations were performed using the parallel flow solver TRACE (Turbomachinery Research Aerodynamics Computational Environment) which is developed at the Institute of Propulsion Technology of the German Aerospace Center (DLR) in cooperation with MTU Aero Engines. As described by [12] turbomachinery flows are modelled and investigated by solving Reynolds-Averaged Navier Stokes (URANS) equations. For turbulence modelling the Wilcox k- $\omega$ turbulence model (see [13]) with extensions by Kato and Launder as described in [14] and for system rotation is applied. No predictions for laminar to turbulent transition were made as the simulations do not use a fine mesh, so that wall functions have to be applied. To obtain a second order accuracy of the spatial discretization, an upwind-based total variation disminishing (TVD) scheme by Roe with a van Leer MUSCL extrapolation is used. As e.g. in case of shock numerical oscillations can occur, a Van Albada flux limiter is used. The steady-state simulation is carried out using a first order implicit Euler backward scheme while the transient simulation uses an implicit second order Euler backward scheme. For inter-row coupling for stationary as well as for time resolved calculations a conservative zonal approach based on flux interpolation with second order accuracy is used.

\section{GRID GENERATION}

One of the main goals is to determine the mixing behaviour of different temperature inhomogeneities at the inlet of the turbine. In this context it shall be examined which inhomogeneities are still detectable at the outlet. The computation of only a part of the turbine would lead to an adjustment of the total number of the passages for each row in order to be able to generate a mesh with only $1 / 6$ of the total passages. This would result in further inaccuracy of the computation. Furthermore, in a preliminary study only one temperature inhomogeneity was included and a steady state simulation was run. The results revealed that, by increasing the geometric gap between the streaks, it is possible to evaluate the mixing characteristic of several cold streaks with different temperature ratios in one simulation. Hence in case of simulating just some passages of the turbine several simulations would be necessary to get the same information level compared to a full annulus computation. Moreover, the circumferential change of the cold streak after passing the whole turbine cannot be clearly predicted and therefore the minimal number of passages cannot be defined. Therefore, instead of performing several calculations with only few passages of the turbine and repeating them with different inhomgeneities at the inlet, one computation of the whole turbine including several local changes of the inlet temperature shall be carried out.

The basis for the structured mesh was a fine grid (Mesh I) using the OCHG-Topology as illustrated in Fig. 1. The mesh size of the design grid would lead to a too high number of nodes and the computation would not be manageable.

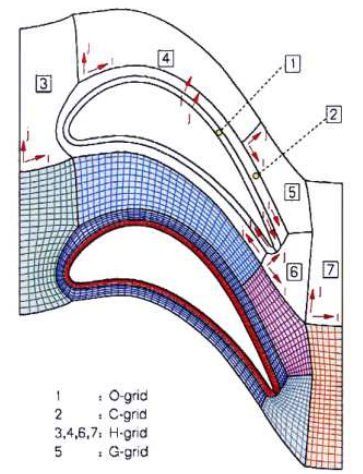

Fig. 1 OCHG-Topology [15]

Therefore Mesh I was coarsened in every direction (i,j,k) resulting in 100 million nodes (Mesh II). Further coarsing reduced the number of nodes to 50 million (Mesh III). For the examination of the mesh quality one passage was computed. As the measurement of the accuracy of the mesh variations the total pressure loss coefficient of the blades which is defined by Eq. (1) was examined.

$$
\zeta=\frac{p_{t o t, 0 \_50 \%}-p_{t o t, 1}(r)}{p_{t o t, 0 \_50 \%}-p_{s t, 1 \_50 \%}}
$$

In Fig. 2 the relative total pressure loss coefficient is compared between the three mesh variations. Mesh II reproduces well the original characteristics. Mesh III however already shows greater differences within spans of $0.7-1$ and $0.1-0.3$. Furthermore the maximal residuals of Mesh III were varying until the range of $10^{-3}$. Hence, Mesh II was selected for the further computations. Regarding the main goals of the study the inaccuracy resulting from the coarsened mesh is acceptable.

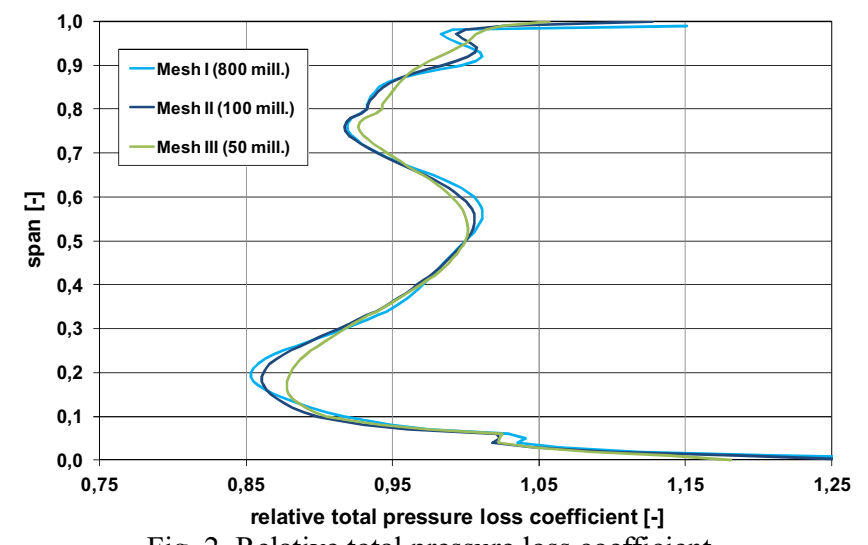

Fig. 2 Relative total pressure loss coefficient

Table 1 gives an overview of the total amount of nodes. Mesh II consists of around 100 million nodes spread out on 9318 Blocks. The nondimensional wall distance $\mathrm{y}^{+}$reaches values of around 30 at the blade surfaces. The meshing for one blade is illustrated in Fig. 3. The complete mesh with every other node for one passage is shown 
in Fig. 4. For a better illustration the stator vanes including the exit guide vane are colored red while the rotor blades are colored green. The figure illustrates well the difference in size between the exit guide vanes and the stages. Hence, it can already be assumed that the wakes of the guide vanes might have a greater influence on the mixing characteristic of the inhomogeneities within the exit nozzle. Furthermore, the number of nodes in circumferential direction in the exit nozzle is decreased. In the upstream direction of the exit guide vanes the mesh density ratio in circumferential direction of the interface between the rotor blades of stage five and the nozzle is 2:1.

Table 1: Mesh-Overview of Mesh II

\begin{tabular}{c|c|c}
\hline & Blocks & Hexa-Elements \\
\hline Stator 1 & 890 & 7.870 .629 \\
Rotor 1 & 970 & 8.293 .888 \\
Stator 2 & 900 & 7.387 .200 \\
Rotor 2 & 990 & 8.540 .928 \\
Stator 3 & 930 & 8.017 .344 \\
Rotor 3 & 870 & 7.811 .904 \\
Stator 4 & 990 & 8.534 .592 \\
Rotor 4 & 850 & 7.349 .440 \\
Stator 5 & 960 & 7.934 .976 \\
Rotor 5 & 890 & 8.196 .544 \\
Outlet & 78 & 1.506 .560 \\
\hline Total & $\mathbf{9 3 1 8}$ & $\mathbf{8 2 . 4 4 4 . 0 0 5}$ \\
\hline
\end{tabular}
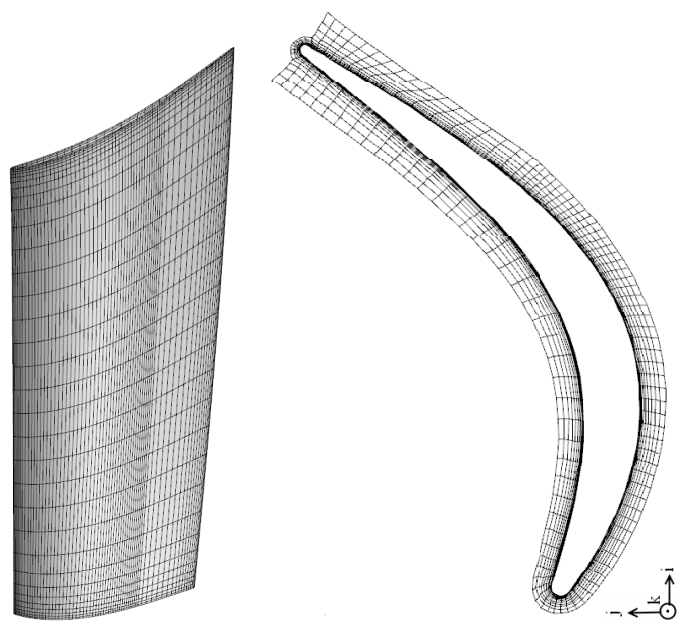

Fig. 3 Meshing of one blade (Mesh II)

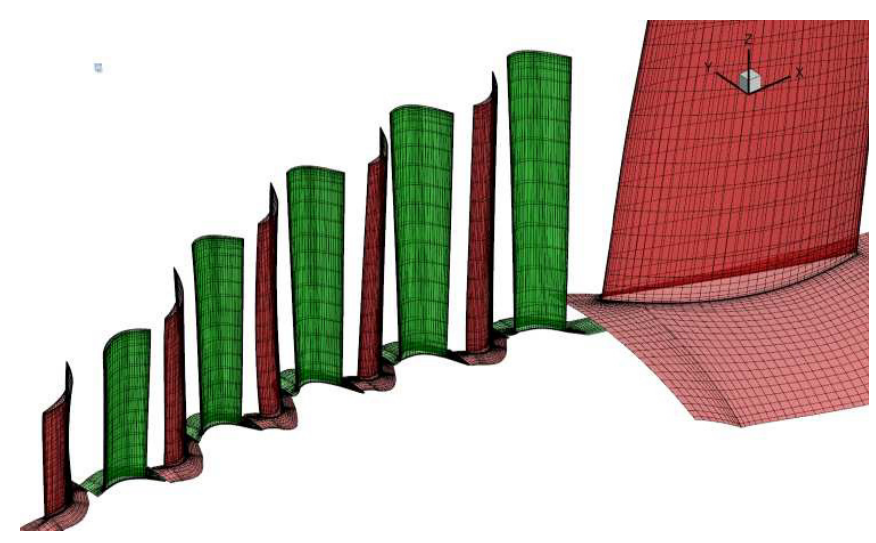

Fig. 4 Meshing of the turbine

\section{STEADY STATE COMPUTATION}

Turbines are often designed to reduce the circumferential velocity at the outlet. For the steady state as well as time resolved computations an operating point with a higher rotational speed than the design point of the turbine was used. The reason is based on the maximal possible deflection in the operating point. As already described one reason for the decision running a whole annulus computation of a turbine was the inaccuracy of the prediction to which extent a particle passing through the turbine will be deflected in circumferential direction. At the operating point with an increased rotational speed the deflection of the rotor against the rotational direction is decreased. Hence the total deflection imposed by the stator vanes in rotational direction will be increased representing the maximal total change in circumferential position. So with the computation a general conclusion on the deflection will be possible. On this basis it will be possible to predict the minimal number of passages in further investigations.

As described by [11] secondary flows induced by hot streaks lead to an increased radial spread of the temperature at the rotor pressure side. Furthermore, the difference between the radius of curvatures between the two sides of the stator blade leads to a radial deflection of the flow in different directions. A hot streak migrates towards the casing at suction side and towards the hub at the pressure side. Thus spanwise deflection of cold streaks are expected. However within this paper as a first step only circumferential deflection of the cold streaks as well as the mixing characteristics shall be investigated. Therefore, to limit the spanwise transport the temperature inhomogeneity shall be varied over the whole span. As described before, in order to examine the effects of several cold streaks in one simulation, the inlet condition of the turbine was modified so that a complex temperature distribution was achieved. The inlet area was divided in 20 equal sized parts according to the number of burners in the combustor. The minimal temperature was defined by the worst case defect, a shutdown of the burner. The remaining differences were distributed logarithmical between the regular inlet conditions and the minimal temperature. The temperature distribution is illustrated in Fig. 5 showing the different temperature ratios which are defined by Eq. (2).

$$
R=\frac{T_{c s}}{T_{\infty}}
$$

The Ratio R_A represents the design temperature at the inlet of the turbine assuming a regular operating combustor. The Ratio R_G represents the corresponding temperature for a worst case defect, a complete shutdown of the burner. Based on the preliminary examination it can be assumed that, due to the great geometric gap, the cold streaks will not interfere with each other. As the temperature decrease results in an increase of the density at the inlet, the total mass flow was increased by $3.7 \%$.

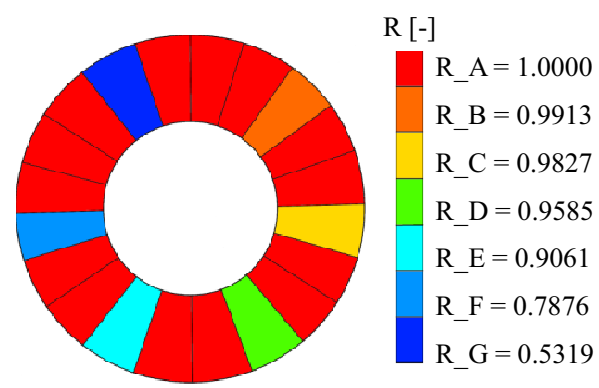

Fig. 5 Temperature ratios at the Inlet

On this basis a steady state simulation was performed using 512 processors and a total of 3840 GB memory. For the simulations a frozen rotor approach was used. The results shall be used as a starting solution for the later performed unsteady full annulus computation of the turbine.

In Fig. 6 the contour plot of the relative temperature over the circumferential and axial direction in $50 \%$ span is illustrated. The 
characteristics at $10 \%$ and $90 \%$ span do not show greater differences to Fig. 6. From the illustration it can be derived that the circumferential position of the temperature inhomogeneities changes only to a small extent. However, as the rotational speed of the calculated point is higher than the design point, the deflection of the stator vanes should not be balanced out by the deflection of the rotor blades. Hence, the circumferential position should change through the turbine. The missing of this effect in the computational results can be mainly explained by the usage of the frozen rotor approach. In this case not the mesh but only the reference system is rotated. Thus, it can be assumed that the time-resolved computation will lead to a slightly different result, showing a deflection of the cold streaks in rotational direction. The computations also show that the cold streaks barely mix with the surrounding flow.

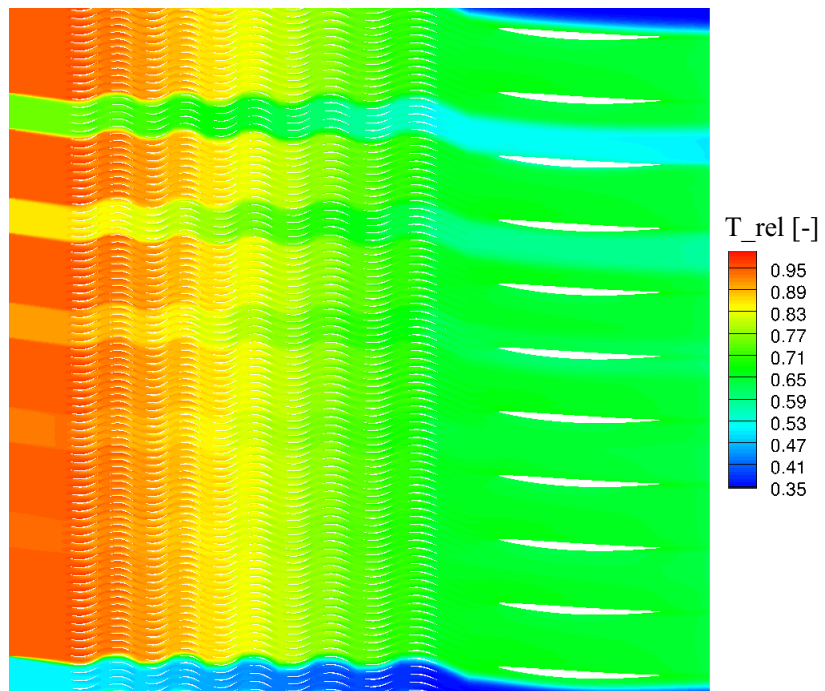

Fig. 6 Contour plot of the relative temperature at $50 \%$ span

Figure 7 illustrates the temperature distribution over the angle in circumferential direction after each stage indicating the different temperature inhomogeneities at $50 \%$ span. The graph shows the described phenomena. The temperature inhomogeneities do not mix out, hence are still detectable at the exit and their circumferential deflection does not exceed $20^{\circ}$.

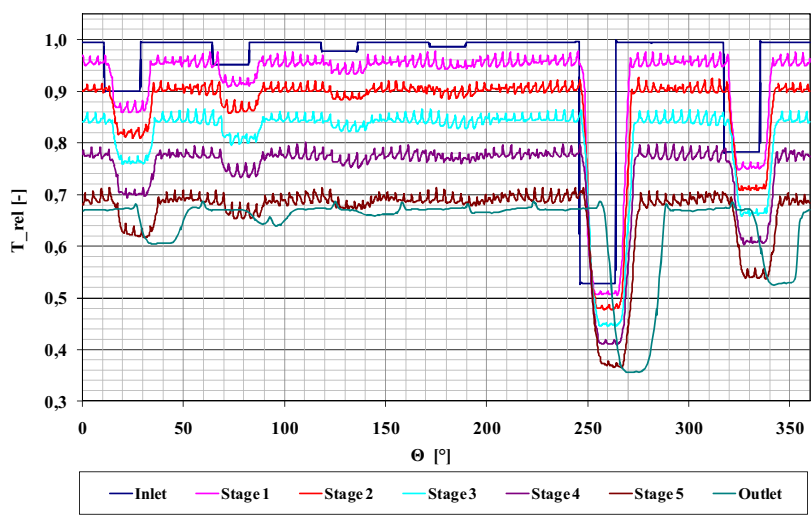

Fig. 7 Deflection of the temperature inhomogenities through the turbine

\section{TIME RESOLVERD FULL ANNULUS COMPUTATION}

With the steady state computation as the starting solution the time resolved simulations were run. For the highest number of passages 20 time steps were performed for one rotor blade passing period, resulting in almost 2000 iterations for one rotation. The inner iterations were set to 10 . Figure 8 shows the temperature contour over the axial and circumferential position at $50 \%$ span. It can clearly be seen, that the deflection increased in comparison to the steady state result. As expected the increased circumferential velocity of the rotor results in a decreased deflection of the flow after the rotor blades. In comparison to the steady state simulations the time-resolved calculations model a more realistic reconstruction of the flow. From the illustration it is also visible that mixing between the cold streaks and the surrounding flow is increased and hence the hottest streaks cannot be clearly detected at the exit. However as shown by the contour of the relative entropy in Fig. 9 even the lowest temperature difference does not completely mix out with the surrounding flow.

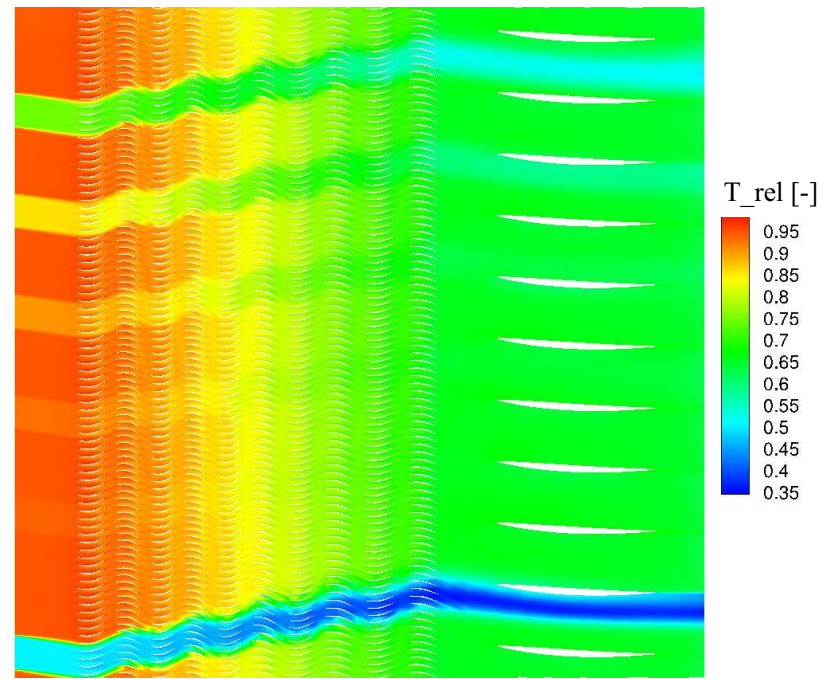

Fig. 8 Contour plot of the temperature at $50 \%$ span

As shown in Fig. 4 and already described, only upwise of the exit guide vanes the mesh density ratio in circumferential direction of the interface between the rotor blades of stage five and the nozzle is $2: 1$. The ratio increases aside of the exit guide vanes, resulting in an unphysical effect which is mixing out the entropy peaks after the rotor blades in the nozzle. As illustrated, the mixing characteristics and deflection of the cold streaks do not change with the position of the cold streaks relative to the exit guide vanes. Hence, the numerical mistake has only a major effect on small inhomogeneities (e.g. rotor wakes) rather than on the whole cold streak.

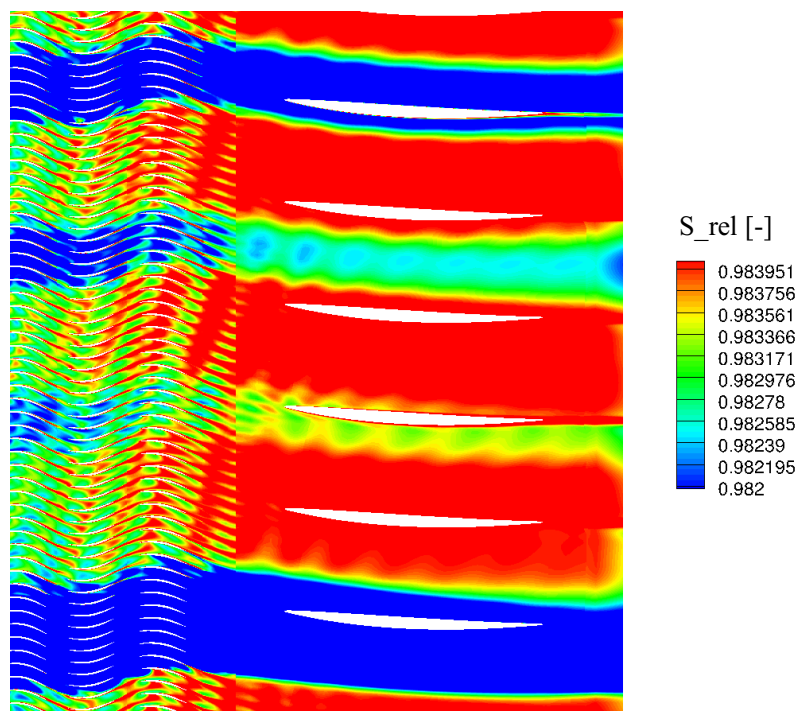

Fig. 9 Contour plot of the relative Entropy at 50\% span of the hottest streak

The overall flow deflection and mixing characteristic does not change with the time, so that the illustration of the results is limited 
here to one time step. However, there are greater changes dependent on the span height.

Figures 10-12 show the temperature distribution over the angle after each stage indicating the different temperature inhomogeneities at $10 \%, 50 \%$ and $90 \%$ span. For the interpretation of the results the whole geometry can be divided into the turbine and the exiting nozzle. The illustrations show that the inhomogeneities mix out with the surrounding flow to a greater extent in the turbine than deriving from the steady-state simulations. Both, the deflection of the circumferential position as well as the mixing increase with the span. The angle varies between $20^{\circ}$ and almost $50^{\circ}$. It can also be seen that even after the fifth stage, the smallest inhomogeneity can still be detected. Hence as already derived from the steady-state results inhomogeneities barely mix out within the blade rows.

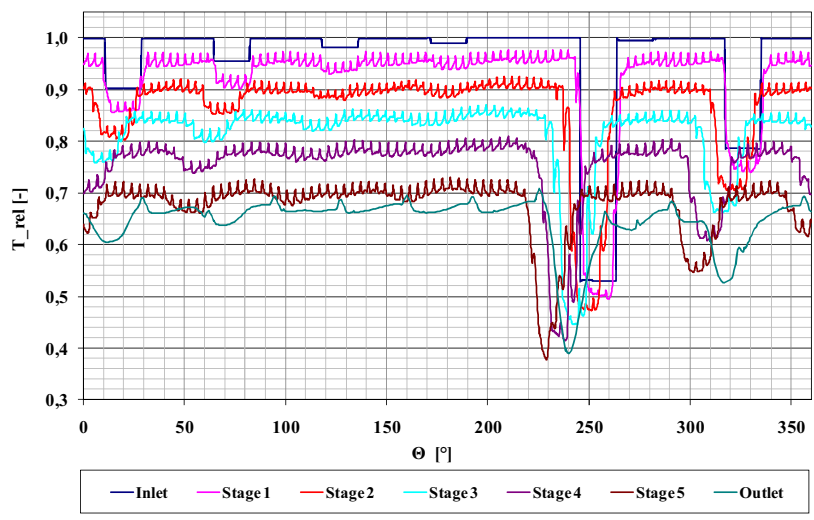

Fig. 10 Relative temperature distribution after each stage over the angle at $10 \%$ span

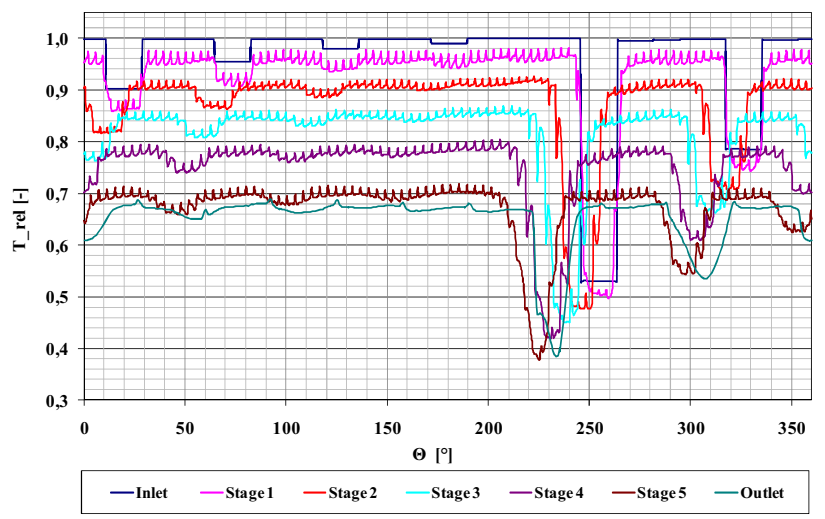

Fig. 11 Relative temperature distribution after each stage over the angle at $50 \%$ span

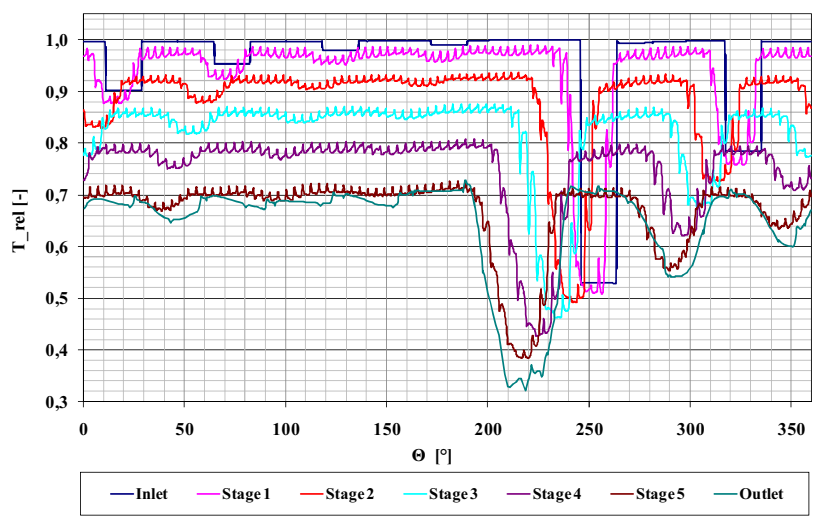

Fig. 12 Relative temperature distribution after each stage over the angle at $90 \%$ span
The characteristic of the flow in the nozzle however changes in comparison to the turbine. The deflection of the flow is against the rotational direction of the turbine and decreases over the span, having a maximum at $10 \%$ span with around $5^{\circ}$ of circumferential angle. Furthermore, it can clearly be seen that the wakes of the exit guide vanes have an extended effect on the temperature distribution. The temperature distribution at the exit of the nozzle, shown in Figures $10-12$ have a characteristical local temperature increase over the whole span caused by the wakes of the exit guide vanes. Based on the mixing effects in the nozzle the two highest temperature inhomogeneities cannot be clearly detected at the exit. In Fig. 13 the relative temperature contour at the exit of the last stage is shown. Despite the wakes from the last stage, all cold streaks are still detectable. However, the streak with the highest temperature ratio is almost completely mixed out. For the four streaks with the lowest temperature ratios a characteristical shape is recognizable. It can be seen that the flow is deflected over the span in the rotating direction due to increased deflection of the flow at higher span.

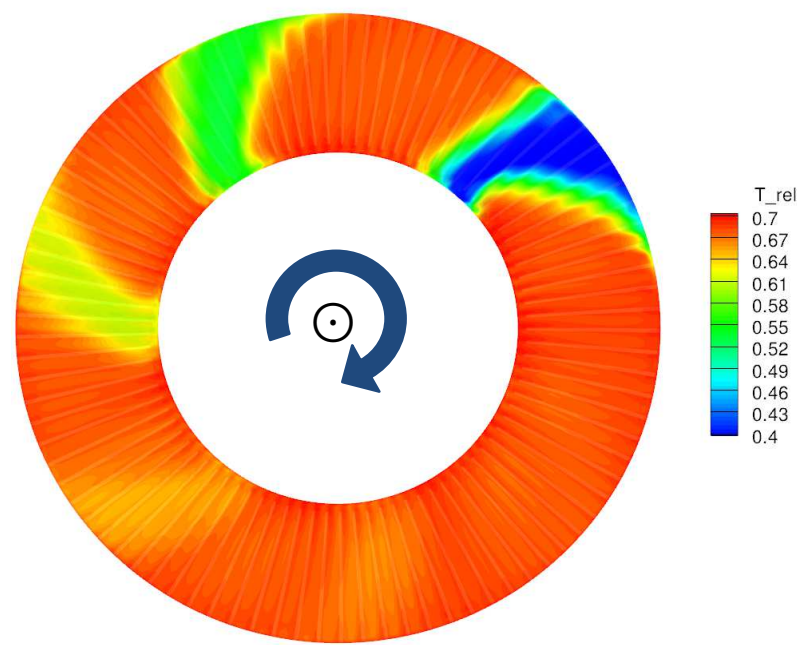

Fig. 13 Temperature contour at the exit of the turbine

As already mentioned, the size of the wakes of the exit guide vanes have a great effect on the mixing of the cold streaks. Hence, in Fig. 14 the relative temperature contour of the nozzle exit is shown.

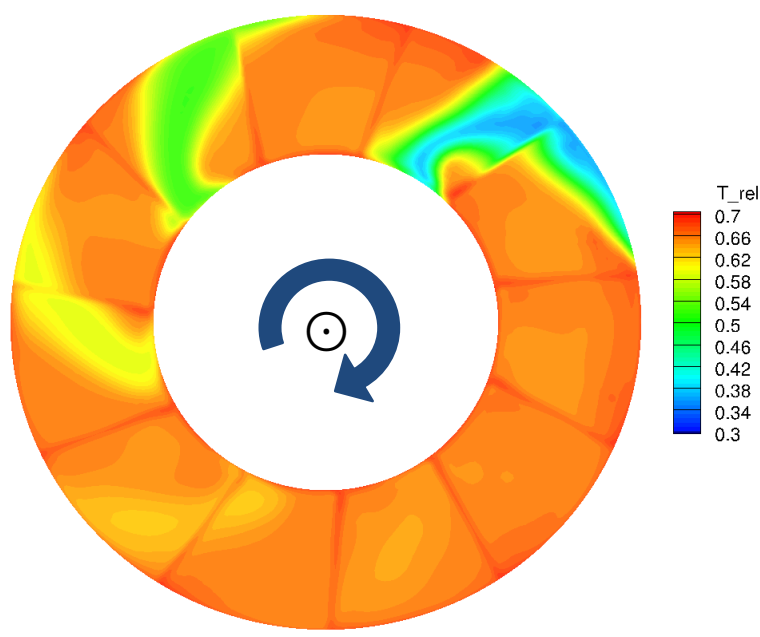

Fig. 14 Temperature contour at the exit of the nozzle

From the illustration, the described local temperature rise of the wakes of the exit guide vanes as well as the increasing flow deflection along the span are clearly visible. Furthermore it is visible that the wakes divide the area of the cold streaks. In Fig. 15 the same view as in Fig. 14 but with a decreased relative temperature 
range is illustrated. Figure 15 shows that against the assumptions derived from Fig. 14 even the cold streak with the highest temperature ratio (R_B) is still not completely mixed out. Hence, a small area with decreased temperature is still visible. As BOS is a highly sensitive optical measurement technique which does not affect the flow it can be assumed that even these small temperature change will be measurable.

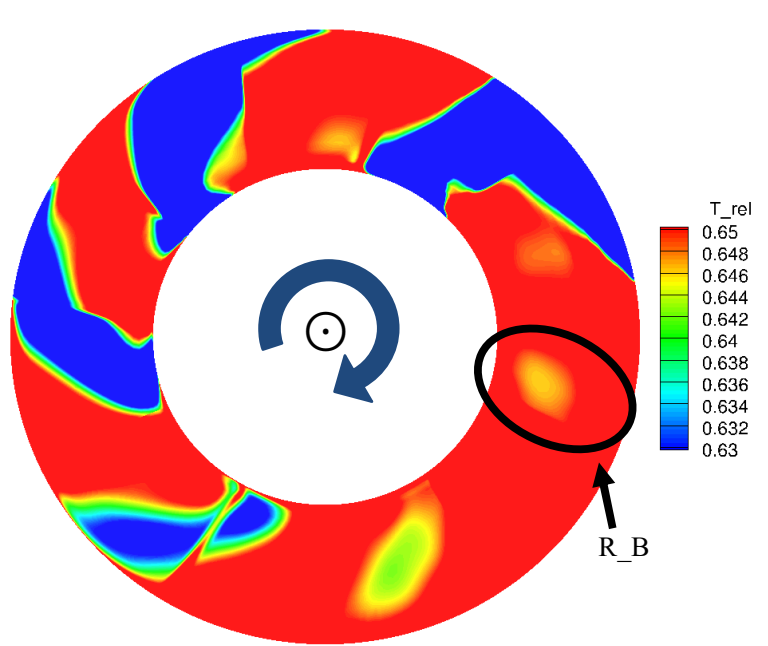

Fig. 15 Temperature contour at the exit of the nozzle with decreased range of temperature contours

To get further information about the mixing behaviour of the inhomogeneities the affected massflow and the average temperature of the cold streaks is analyzed. The affected massflow was defined as the flow which has, considering each cold streak separately, a decreased temperature in comparison to the surrounding, unaffected flow. For the affected massflow of the cold streaks the area averaged temperature was calculated. For the investigation planes at the inlet as well as the outlet of the whole turbine and between the blades and vanes have been evaluated. Figure 16 illustrates the ratio of the average temperature of the cold streaks and the whole turbine over the axial position. The cold streaks are named in correspondence with the temperature ratios at the inlet of the turbine as defined and illustrated in Fig. 5.

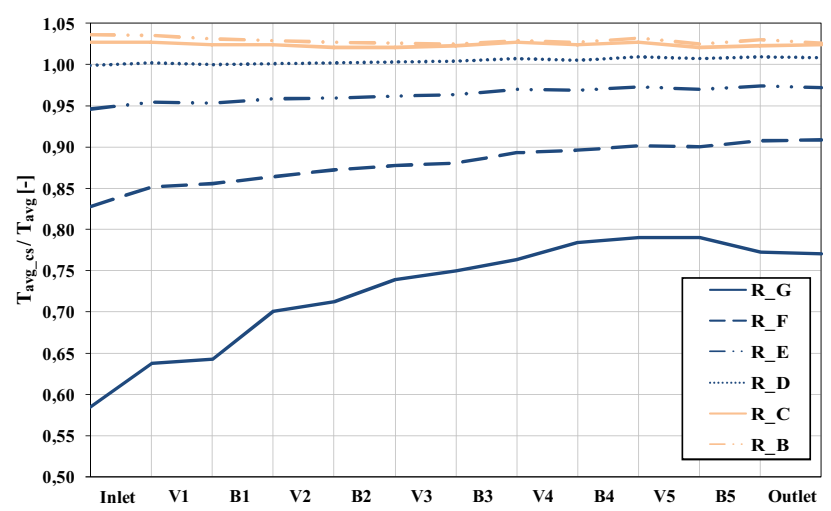

Fig. 16 Normalized average temperature of the cold streaks over the axial position of the turbine

From the illustration it can be seen, that especially the streaks R_G and $R$ F with a low temperature ratio and great temperature difference to the surrounding flow already mix in the inlet before entering the turbine. The streaks with higher ratio do not show the same mixing behaviour. Hence, it can be concluded that the main mixing effect is the high temperature difference. As expected it is also visible that mixing increases when the temperature difference between the cold streak and the surrounding flow is increased.
However, the temperature of the cold streaks is not the only dimension which should be evaluated. A cold streak with a high temperature difference but small massflow might have a much lower effect than a cold streak with high massflow but low temperature difference. By defining the nondimensional turbine mixing factor TMF as defined in Eq. (3) an approach for the analysis of the mixing behaviour is derived.

$$
T M F=\frac{\left(T_{a v g_{-} c s}-T_{a v g_{-} \infty}\right) \cdot \dot{m}_{c s}}{\dot{m}_{\infty}}
$$

The TMF does not only take the average temperature of the cold streak in comparison to the surrounding flow into account but also the massflow and enables therefore a complete assessment how fast the inhomogeneities mix out with the surrounding flow and whether they can be detected at the outlet. Streaks with a high absolute value will have a greater effect on the surrounding flow. The simulations have shown that the cold streaks do not interfere with each other. Hence, for the calculation of the TMF it is assumed that, excluding the considered cold streaks, the rest of the turbine is without defects. In this way it is possible to evaluate the effect of each cold streak separately and make a statement on how fast they mix out. In Fig. 17 the TMF of the cold streaks is illustrated over the axial position in the turbine. The change of the factor equals the mixing between the inhomogeneity and the surrounding flow.

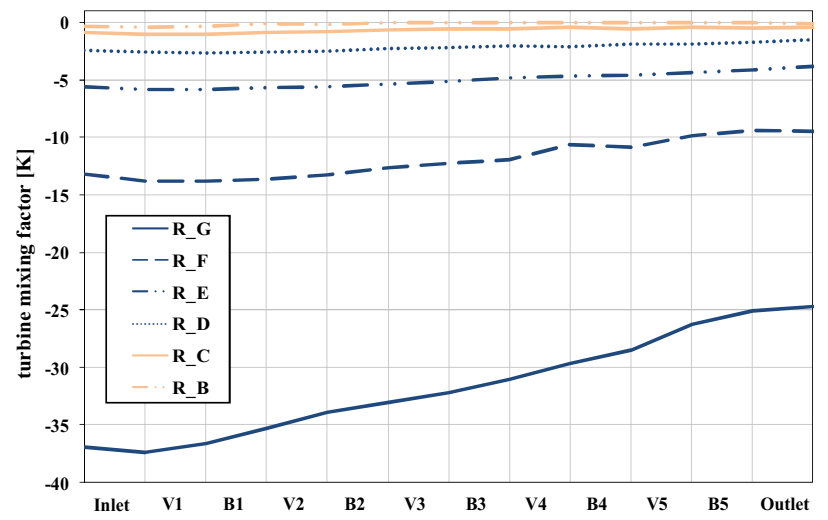

Fig. 17 Turbine mixing factor (TMF) of the inhomogenities over the axial position of the turbine

As expected the low temperature ratios have a much higher possible effect on the turbine than the high ratios. It can also be seen that the mixing effects increase with the temperature difference. Furthermore, based on the gradient of the TMF of the ratios R_F and R G it can be assumed that mixing effects are increased by the vanes and blades in comparison to the unguided flow at the inlet and outlet. With the defined TMF in subsequent studies a minimal value can be determined which will be detectable in the exhaust gas stream and hence can be measured by BOS at the outlet. On this basis it will be possible to make an assessment whether the effect of a defect on the local temperature is high enough to be detected in the exhaust gas stream.

\section{CONCLUSIONS AND OUTLOOK}

The simulations show a great difference between steady-state and unsteady computations. While the steady-state computations show only minimal change of the circumferential position of the cold streaks through the turbine, the time-resolved simulations can reproduce a realistic deflection. Changes to the deflection and mixing effects within one blade passing period are negligible. The deflection also demonstrates that full annulus computations are not always necessary as even the maximal deflection angle does not exceed $50^{\circ}$.

The time-resolved simulations show that cold streaks are deflected 
to a greater extent close to the outer casing of the turbine than near the hub. The derived turbine mixing factor enables an integral assessment of the mixing characteristic of the cold streaks over the whole turbine and a statement whether an effect can still be detected at the outlet. In this context it is demonstrated that even cold streaks with very high temperature ratios are still detectable at the exit of the nozzle. Hence it can be assumed that defects causing local temperature changes within the hot gas path can be detected with the BOS method in the exhaust jet of the engine.

In a next step, further calculations of the exhaust gas stream will be carried out to investigate how the temperature inhomgeneities mix out in the jet. Until now, the cold streaks were established over the whole span. In a subsequent step, small temperature inhomogeneities with a small massflow ratio shall be examined. In this context, the physics behind the mixing of the cold streaks with the surrounding flow will be investigated closer.

\section{ACKNOWLEDGMENTS}

The authors thank the North-German Supercomputing Alliance (HLRN) for the provided computational resources. The paper presents research results of project A3 conducted within the Collaborative Research Centre 871 which is funded by the Deutsche Forschungsgemeinschaft. Furthermore the authors want to thank Thomas Kippel for the contribution made with his diploma thesis.

\section{REFERENCES}

[1] Goldhahn, E.; Seume, J. R., 2007, "The Background oriented schlieren technique: sensitivity, accuracy, resolution and application to a tree-dimensional density field", Experiments in Fluids, Vol. 43, Issue 2-3, pp. 241-249

[2] Goldhahn, E.; Alhaj, O.; Herbst, F.; Seume, J. R., 2009 , "Quantitative Measurements of Three-Dimensional Density Fields Using the Background Oriented Schlieren Technique", Nitsche, W.; Dobriloff, C.: Imaging Measurement Methods for Flow Analysis, Springer, Berlin, pp.135-144

[3] Alhaj, O., Seume, J. R., 2010, "Optical Investigation of Profile Losses in a Linear Turbine Cascade", Proceedings of GT2010 ASME Turbo Expo 2010: Power for Land, Sea and Air, GT2010-23166

[4] Belamri, T., Braune, A., Cornelius, C., Galpin, P., 2005, "CFD Analysis of a 15 Stage Axial Compressor Part I: Methods", Proceedings of GT2005 ASME Turbo Expo 2005: Power for Land, Sea and Air, GT2005-68261

[5] Belamri, T., Braune, A., Cornelius, C., Galpin, P., 2005, "CFD Analysis of a 15 Stage Axial Compressor Part II: Results", Proceedings of GT2005 ASME Turbo Expo 2005: Power for Land, Sea and Air, GT2005-68262

[6] Herrick, G. P., Hathaway, M. D., Chen, J.-P., 2009, "Unsteady Full Annulus Simulations of a Transonic Axial Compressor Stage", 47th Aerospace Sciences Meeting, AIAA-2009-1059

[7] Im, H.-S., Chen, X., Zha, G.-C., 2010, "Detached Eddy Simulation of Unsteady Stall Flows of a Full Annulus Transsonic Rotor", Proceedings of GT2010 ASME Turbo Expo 2010: Power for Land, Sea and Air, GT2010-23465

[8] Röber, T., Kügeler, E., Weber, A., 2010, "Investigation of Unsteady Flow Effects in an Axial Compressor Based on Whole Annulus Computations", Proceedings of GT2010 ASME Turbo Expo 2010: Power for Land, Sea and Air, GT2010-23522

[9] Qureshi, I., Beretta, A., Povey, T., 2011, "Effect of Simulated Combustor Temperature Nonuniformity on HP Vane and End Wall Heat Transfer: An Experimental and Comuptational Investigation", 2011, ASME Journal of Engineering for Gas Turbines and Power, Vol. 133

[10] Qingjun, Z., Fei, T., Huishe, W., Jianyi, D., Xiaolu, Z., Jianzhong, X., 2008, "Influence of Hot Streak Temperature Ratio on Low Pressure Stage of a Vaneless Counter-Rotating Turbine", ASME Journal of Engineering for Gas Turbines and Power, Vol. 130

[11] Basol, A. M., Jenny, P., Ibrahim, M., Kalfas, A., I., Abhari, R.
S., 2011, "Hot Streak Migration in a Turbine Stage: Integrated Design to Improve Aerothermal Performance", ASME Journal of Engineering for Gas Turbines and Power, Vol. 133

[12] Biester, M. H.-O., Mueller, L., Seume, J. R., Guendogdu, Y., 2011, "Time-Resolved Numerical Investigation of the Interaction of Labyrinth Seal Leakage and Main-Flow in a 1.5-Stage LP Turbine", Proceedings of GT2011 ASME Turbo Expo 2011 Power for Land, Sea and Air

[13] Wilcox, D. C., 1998. Turbulence Modeling for CFD, 2nd ed. DCW Industries, Inc.

[14] Kozulovic, D., Röber, T. K., Kügeler, E., and Nürnberger, D., 2004. "Modifications of a two-equation turbulence model for turbomachinery fluid flows".

[15] Weber, A. 2007, "3D structured grids for multistage axial turbomachines and linear cascades", Version 4.2, Cologne, DLR - Institute of Propulsion Technologies 